\title{
VIRILIZING ADRENOCORTICAL TUMOR: A CASE REPORT.
}

\section{Maraver-Selfa S, Cornejo-Pareja I, Díaz-Perdigones C, Mancha-Doblas I, Tinahones FJ.}

Endocrinology and Nutrition Department. Virgen de la Victoria Hospital, Málaga, Spain.

\section{INTRODUCTION}

Adrenal masses are among the most frequent tumours in humans (ACT). A vast majority of these tumours are benign (ACAs). Only a small subset of adrenal masses are malignant adrenocortical carcinomas (ACCs). Tumour size, tumour weight, hormonal function and pathologic criteria are useful clinicopathological criteria that can result in accurate diagnosis of most ACCs and ACAs.

\section{CASE REPORT}

31 years old woman, without relevant previous history. She had long evolution hirsutism, acné and androgenetic alopecia, with gradual worsening (SAHA syndrome). Menarche: 9 years old. She had always had irregular menstruations, and amenorrhea periods, alternating with polymenorrhea. She was treated with oral contraceptives previously.
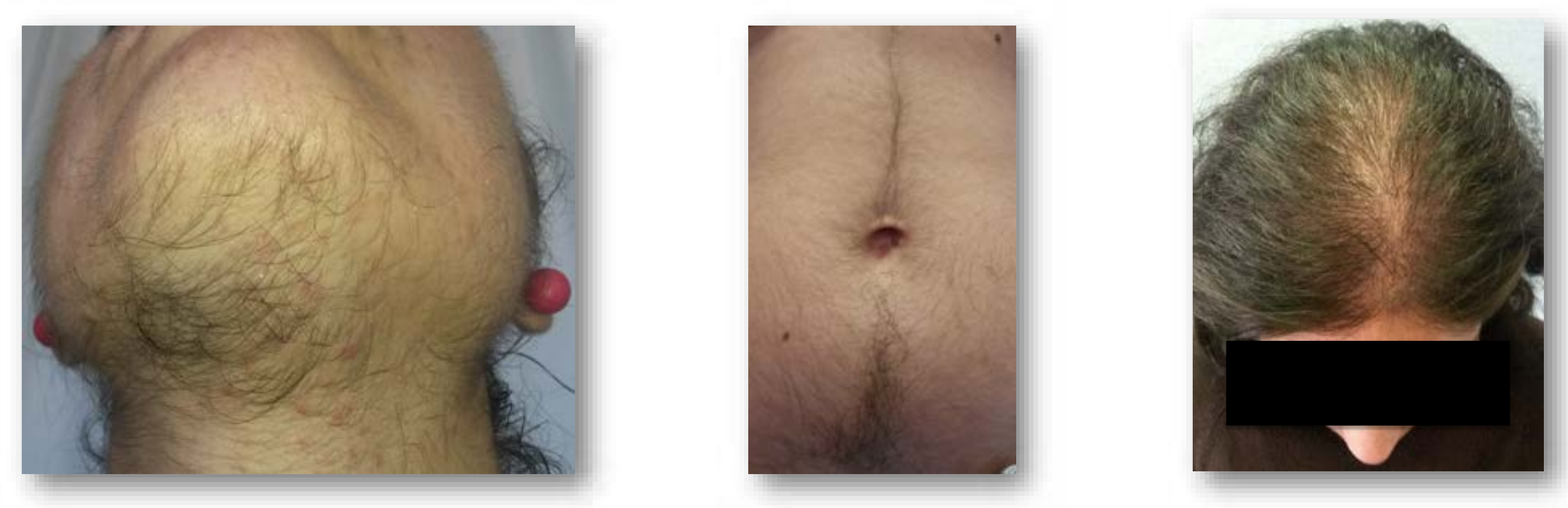

\section{Physical examination: $50 \mathrm{Kg}, 148 \mathrm{~cm}, \mathrm{BMI} 22,8 \mathrm{~kg} / \mathrm{m2}$, Blood pressure $115 / 85 \mathrm{mmHg}$, Ferriman scores 25 points.}

\section{Complementary Studies}

\section{HORMONAL STUDY:}

TSH $0.57 \mu \mathrm{UI} / \mathrm{mL}$, free-T4 $0.76 \mathrm{ng} / \mathrm{dL}, \mathrm{FSH} 3.85 \mathrm{mUI} / \mathrm{mL}$, LH $4.28 \mathrm{mUl} / \mathrm{mL}$, Prolactin $18.6 \mathrm{ng} / \mathrm{mL}$, Testosterone $2.93 \mathrm{ng} / \mathrm{mL}$ (<1.2), DHEA-S $1.825 \mu \mathrm{g} / \mathrm{dL}(<560), 17-\mathrm{OH}$ progesterone $5.14 \mathrm{ng} / \mathrm{mL}$, Basal cortisol $21.2 \mu \mathrm{g} / \mathrm{dL}(<25)$, ACTH $1 \mathrm{pg} / \mathrm{mL}$, Androstendione $12.4 \mathrm{ng} / \mathrm{mL}(<3.5)$, overnight dexamethasone suppression test $9.8 \mu \mathrm{g} / \mathrm{dL}$, UCL $381 \mu \mathrm{g} / 24 \mathrm{~h}$ (diuresis $1.700 \mathrm{ml} / 24 \mathrm{~h}, \mathrm{Cr} .43 \mathrm{mg} / \mathrm{dl}$ ).

\section{MAGNETIC RESONANCE IMAGING (MRI):}

Right adrenal mass $(6.1 \times 5.6 \mathrm{~cm})$, with inferior vena cava mark, heterogeneous intensity, isointense in $\mathrm{T} 1$ and lightly hyperintense in $\mathrm{T} 2$.
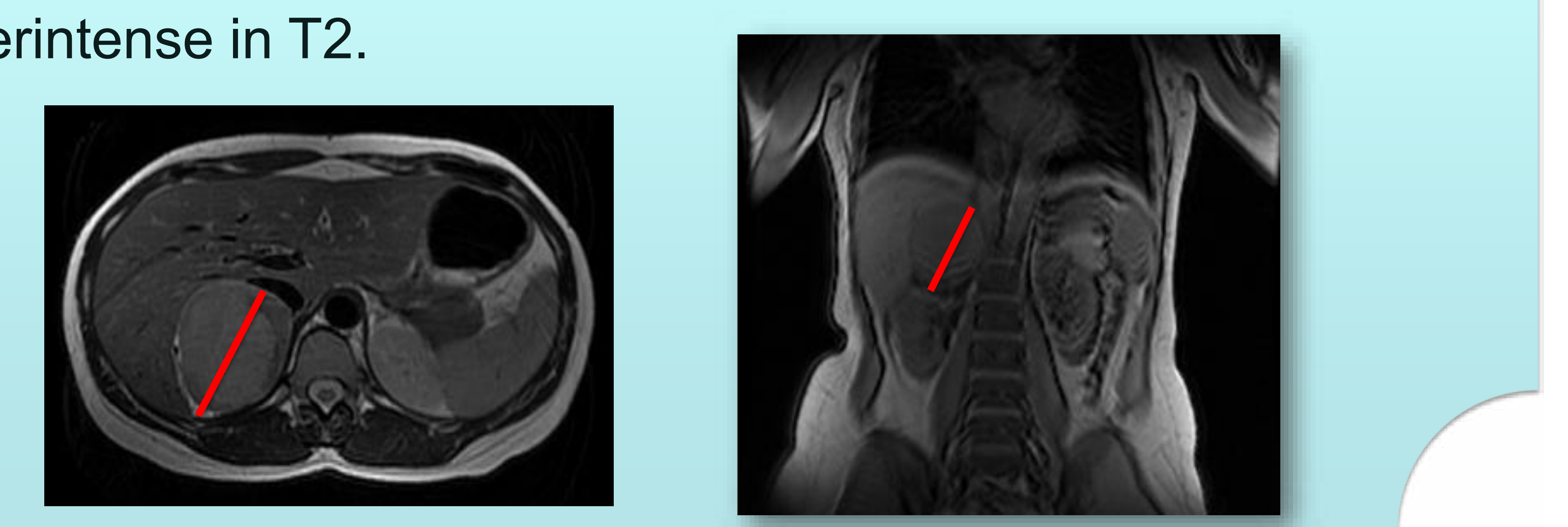

\section{We decided right adrenalectomy.}

\section{HISTOPATHOLOGY:}

$6,8 \mathrm{~cm}$ and $120 \mathrm{~g}$ homogeneous lesion, smooth surface, compatible with corticoadrenal adenoma.

Post-surgery she began hydrocortisone replacement therapy and remained stable. She spontaneously menstruated and losted $4 \mathrm{Kg}$.

BIOCHEMISTRY: Testosterone<0.01ng/mL, DHEA$\mathrm{S}<15 \mu \mathrm{g} / \mathrm{dL}, 17-\mathrm{OH}$ Progesterone $0 \mathrm{ng} / \mathrm{mL}$, Basal cortisol $4,9 \mu \mathrm{g} / \mathrm{dL}, \mathrm{ACTH} 1 \mathrm{pg} / \mathrm{mL}$, Androstendione $0,1 \mathrm{ng} / \mathrm{mL}$ y UCL $91 \mu \mathrm{g} / 24 \mathrm{~h}$.

Now treatment is being reduced gradually, according to clinical evolution.

\section{CONCLUSIONS}

$\wp$ It is difficult to distinguish between a benign and malign ACT, even with anatomo-pathology diagnosis. There are no good histologic criteria to distinguish adenoma from carcinoma.

$\wp$ The best way to determine malignancy is the clinical evolution.

$\wp$ In our patient, the fast androgens reduction post-surgery is an indicator of surgical success. Clinical evolution and biochemistry determine initial pathology report. 